\title{
Annotations
}

\section{Who needs a brain scan?}

Over the last 10 years computed tomography scanning of the brain has had a remarkable effect on paediatric neurological practice. Pneumoencephalography is virtually a thing of the past, and angiography has now only a limited place. Since the review by Day and others of the use of scanning in childhood the quality, accuracy, and availability have improved greatly. ${ }^{1}$

Scanning of the brain is particularly helpful in acute illnesses-for example, vascular accidents, or if there is raised intracranial pressure, or coma. In the presence of progressive neurological signs it may identify or exclude the diagnosis of a space occupying lesion, hydrocephalus, and some of the degenerative diseases. The indications are less certain in patients with chronic diseases, and I wish to consider four common clinicàl problems: epilepsy, cerebral palsy, mental retardation, and headache.

These sections can only give guidelines and are, of course, to some extent affected by the accessibility and availability of scanning, whether or not an anaesthetic is required, and the degree of anxiety felt by the family and the doctor.

\section{Epilepsy}

There is good documented evidence that computed tomography in otherwise neurologically normal children with primary generalised epilepsy yields little useful information. ${ }^{2}$ The yield of abnormal scans is somewhat more substantial in children with focal (partial) epilepsy and a focus on the electroencephalogram (EEG). In several series the detection of abnormalities is greater in simple partial seizures than in complex partial seizures. ${ }^{3}$ In both these groups, however, the likelihood of finding a tumour or resectable surgical lesion is very small, especially in the pre-pubertal child.

Routine computed tomography should not be necessary in otherwise normal children with primary generalised seizures, benign rolandic epilepsy, adolescent myoclonic epilepsy, and typical absence attacks, ${ }^{4}$ and it is unlikely that a lesion would be missed. The yield of abnormalities, though they are not necessarily clear primary diagnoses, is significantly higher in children with infantile spasms $(75 \%$ in cases associated with mental retardation) ${ }^{5}$ and myoclonic astatic epilepsy (Lennox syndrome).
Cerebral atrophy, periventricular calcification in tuberous sclerosis, agenesis of the corpus callosum, and cystic lesions are more common findings.

There can be difficulties in interpreting the scans, and it is important that contrast enhancement should be used, otherwise certain changes can be missed. Transient changes may occur possibly due to local vascular phenomena. ${ }^{6}$ Opaque or lucent lesions may be seen which may mimic gliomas and occasionally, if the scanning is performed early in the course of a disease, a positive finding may be missed. One should be prepared to re-scan if the clinical picture warrants it. This is especially true in children if the epilepsy is progressive, resistant, or appears to deteriorate or change (whatever its nature).

Computed tomography will clearly show cysts, or tumours, and macroscopic changes, but will not necessarily show small gliotic areas or cytoarchitectural changes. It may well be that in the long term magnetic resonance imaging will conclusively show these more clearly. ${ }^{7}$

\section{Mental retardation}

As with generalised epilepsy, routine brain scanning in mental retardation of unknown aetiology is unlikely to give helpful diagnostic results. Lingham et al showed that where a scan is positive in a case of mental retardation, it is often not specific (general or focal atrophy) and would not add to diagnostic, prognostic, or genetic information. ${ }^{8}$ Even with the many mental retardation syndromes associated with congenital anomalies, only a small percentage have a specific computed tomogram which is diagnostic.

Computed tomography is more likely to lead to useful results when a primary malformation of the central nervous system is suggested by cranial size and shape. Macrocephaly may show hydrocephalus or megalencephaly. Microcephaly may show polymicrogyria or lissencephaly, and a midline malformation may show holoprosencephaly. When epilepsy is combined with retardation the positive yield is higher, as it is if epilepsy is combined with a visual deficit-for example, septo-optic dysplasia associated with optic nerve hypoplasia.

Bergstrom et al suggested that $20 \%$ of children with neurodevelopmental disorders had some 
abnormality, but most of the findings were minorfor example, some loss of substance of the brain with mild ventricular dilatation. ${ }^{9}$ These findings are difficult to interpret as we still do not know the whole range of scan findings in normal children. The children who had abnormal scans were more likely to have had a seizure.

\section{Cerebral palsy}

In many cases of cerebral palsy the aetiology is not clear or unknown. A scan might help to elucidate the nature of the problem and the extent, but cannot usually date the onset of the problem precisely (which might be important from the medico-legal standpoint). In many cases of symmetrical spasticity the findings on computed tomography are normal. In other cases spastic diplegia may be associated with ventricular dilatation and periventricular leucomalacia. ${ }^{10}$ In dystonic tetraparesis an atrophic brain with ventricular dilatation may be shown. In hemiparesis there is the highest yield of abnormal findings, most commonly a cortical infarct. Occasionally in ataxic states hypoplasia or maldevelopment of the cerebellum is shown.

No sign of abnormality on computed tomography was found by Taudorf et al to be diagnostic of a specific type of cerebral palsy, but hemiatrophy was nearly always seen in patients with hemiplegia. ${ }^{10}$ There was specific atrophy of the cella media in some cases with paraplegia, and it was postulated that this might represent the result of microinfarction in the periventricular area in premature children. A similar suggestion was made by Barth et al, who found that there was a specific pattern of bilateral cortical and subcortical damage in the central regions which was compatible with birth asphyxia. ${ }^{11}$

Thus although computed tomography may be abnormal in most cases of cerebral palsy, the findings are not usually specific enough to be helpful. Scanning is likely to be most valuable in delineating the severity of the lesion associated with hemiparesis and may give a clue to the origin of the problem in some ataxic syndromes. In some cases of diplegia, computed tomography together with other information may help date the origin of the problem.

\section{Headache}

Many parents are concerned about the possibility of a tumour as the cause of a child's headache. With isolated headache as the sole symptom in the absence of neurological findings or signs of raised intracranial pressure, important intracranial disease is unlikely to be shown by scanning. Even in adults where the likelihood of disease is higher, only $7 \cdot 5 \%$ of one large series had unsuspected disease, of which one third were tumours. ${ }^{12}$ In a study of adults with classic migraine only one of 435 patients had an important abnormality, ${ }_{13}^{13}$ and Prensky et al have stated that radiological investigation of children with migraine is unrewarding. ${ }^{14}$ Transient changes have been reported in the scans of children with classic migraine which were at first mistaken for arteriovenous malformations. ${ }^{15}$ It is likely that these changes, seen in the acute phase, represented changes in the permeability of the blood brain barrier. Adults with migraine may show a degree of atrophy when they have had migraine for a number of years, but the importance of this is not known. ${ }^{16}$ Unless recurrent headaches have an unusual pattern, are getting progressively worse, or are associated with neurological signs, computed tomography is not really justified.

The cost of scanning, including perhaps transport to special centres and anaesthesia, is appreciable. The investigation should be performed when a diagnosis, particularly one which will affect management, is likely to be made. It must also be realised that a normal scan does not necessarily exclude an underlying pathological process. When there is no real clinical indication, as in generalised epilepsy or migraine, we should resist the pressures of parents (and doctors) to perform this examination, although occasionally I suspect many of us have given in.

\section{References}

1 Day RE. Thomson JLG, Schutt WH. Computerised axial tomography and acute neurological problems in childhood. Arch Dis Child 1978:53:2-11.

2 Aicardi J, Murnaghan K. Gandon Y, Barton J. Efficacité de la tomodensitometric dans les epilepsies de l'enfant: Problèmes posés par son utilisation. J Neuroradiol 1983;10:127-9.

${ }^{3}$ Guberman A. The role of computed cranial tomography (CT) in epilepsy. Can J Neurol Sci 1983;10:16-21.

${ }^{4}$ Aicardi J. Epilepsy in Children. New York: Raven Press, 1986. (International Review of Child Neurology Series.)

5 Lingham S, Kendall BE. Computed tomography in non-specific mental retardation and idiopathic epilepsy. Arch Dis Child 1983:58:628-9.

6 Jayakumar PN, Taly AB, Mohan PK. Transient computerised tomographic (CT) abnormalities following partial scizures. Acta Neurol Scand 1985;72:26-9.

7 Roach ES, Smith T, Terry CV, Riela AR, Laster DW. Magnetic resonance imaging in pediatric neurologic disorders. $J$ Child Neurol 1987:2:111-6.

${ }^{x}$ Lingham S, Read S, Holland IM, Wilson J, Brett EM, Hoare RD. Value of computerised tomography in children with non specific mental subnormality. Arch Dis Child 1981;57: 381-3.

" Bergstrom K, Bille B, Rassmussen F. Computed tomography of the brain in children with minor neuro-developmental disorders. Neuropediatrics 1984;15:115-9.

16) Taudorf K, Melchior JC, Pedersen H. CT findings in spastic 


\section{Green}

cerebral palsy: clinical, aetiological and prognostic aspects. Neuropediatrics 1984;15:120-4.

11 Barth PG, Valk J, Olislagers-de Slegte R. Central corticosubcortical pattern on CT in cerebral palsy. Its relevance to asphyxia. J Neuroradiol 1984;11:65-71.

12 Baker HL Jr. Cranial CT in the investigation of headache: cost-effectiveness for brain tumours. J Neuroradiol 1983;10: $112-6$.

13 Cuetter AC, Aita JF. CT scanning in classic migraine. Headache 1983;23:195.

14 Prensky AL, Sommer D. Diagnosis and treatment of migraine in children. Neurology 1979;29:506-10.
15 Alvarez-Cermeno JC, Gobernado JM, Freije R, Zaragoza E, Gimeno A. Cranial computed tomography in pediatric migraine. Pediatr Radiol 1984;14:195-7.

${ }^{16}$ du Boulay GH, Ruiz JS, Rose FC. Stevens JM, Zilkha KJ. CT changes associated with migraine. American Journal of Neuroradiology 1983;4:472-3.

S H GREen

The Children's Hospital, Birmingham B16 8ET 